\title{
Practice change amidst the COVID-19 pandemic: Harnessing the momentum for expanding telehealth in transplant
}

To the Editor:

On March 17, 2020, the Centers for Medicare \& Medicaid Services invoked sweeping expansions to the nation's telehealth capacity, authorizing providers to bill for telehealth visits with Medicare beneficiaries at cost parity to in-person visits, eliminating deductibles, and waiving penalties for non-HIPAA compliant communication platforms. ${ }^{1}$ While issued on a temporary and emergency basis, this policy provides an opportunity for the transplant community to explore permanent telehealth infrastructure.

Before the COVID-19 pandemic, our center's long-term goal was to increase utilization of telehealth to $20 \%$ of patient visits over 5 years. COVID-19 has forced us to adapt rapidly. In 2019, we performed 5 telehealth visits per month-in March 2020 alone we performed 184, and have had over 475 telehealth visits in April. We moved the majority of our low-acuity patient visits to video or phone and are working on transitioning required transplant education classes to an interactive, virtual format. During the rapid expansion, we have been stressed by network traffic, availability of compatible technology, and billing concerns, yet we are overcoming these barriers and have made lasting progress through a team-based approach to patient and provider education and technical support.

Long-term transplant telehealth plans at our institution were motivated by evidence that transplant telehealth programs may reduce costs, shorten time to initial evaluation and waitlist placement, improve quality of life, and decrease readmissions following transplant. ${ }^{2,3}$ While these long-term goals still exist, the rapid roll-out has demonstrated additional benefits. Our video care teams are able to frequently check-in on patients with acute medical and surgical issues and reinforce education on challenges like polypharmacy or low health literacy. Anecdotally, patients have been enthusiastic participants and have experienced no known adverse events. We are learning and honing best practices each day but will need to be rigorous in our interpretation of data and mindful of barriers.

As with any change in care delivery, telehealth services must not create or further disparities for the most vulnerable populations. Utilization of telehealth requires at a minimum access to a smartphone and/or Internet. Demographic factors including education, age, language, and culture may all impact telehealth accessibility. At the same time, some of the greatest disparities in the transplantation process occur with waitlist access. Distance from a transplant center has been associated with increased mortality. ${ }^{4}$ If implemented carefully, telehealth may increase access to our rural patients and reduce travel-related time and financial costs. There is also evidence that implementing telehealth measures focused on patients of lower socioeconomic status, creating tools accessible across broad ranges of literacy, and aiming for universal access to technology may allow telehealth expansion to be a solution rather than a barrier to access. ${ }^{5}$

As we move past COVID-19, our department is committed to advancing our infrastructure for telemedicine-based transplantation care. A crisis is an impetus for transformative change, and the current pandemic should be seen as an opportunity to move telehealth forward. We can use this sudden change as a stimulus to discard outdated policies and innovate for the good of our patients.

\section{CONFLICT OF INTEREST}

None declared.

$$
\begin{array}{r}
\text { Keli Siqueiros Santos-Parker }{ }^{1} \text { iD } \\
\text { Jessica Renee Santos-Parker }{ }^{1} \text { iD } \\
\text { Alexandra Highet }{ }^{1} \text { iD } \\
\text { John Richard Montgomery }{ }^{2} \text { iD } \\
\text { Glenn Kham Wakam }{ }^{2} \text { iD } \\
\text { Christopher J. Sonnenday }{ }^{2} \text { iD } \\
\text { Seth A. Waits }{ }^{2} \text { iD } \\
{ }^{2} \text { Section of Transplantation, Department of Surgery, University } \\
\text { of Michigan, Ann Arbor, MI } \\
\text { 1 University of Michigan Medical School, Ann Arbor, MI } \\
\text { Correspondence } \\
\text { Seth A. Waits, Department of Surgery, Section of } \\
\text { Transplantation, University of Michigan, Ann Arbor, } \\
\text { Michigan, USA. } \\
\text { Email: waitss@med.umich.edu }
\end{array}
$$

\section{ORCID}

Keli Siqueiros Santos-Parker iD https://orcid. org/0000-0002-2773-9775

Jessica Renee Santos-Parker (iD https://orcid.

org/0000-0001-5625-9968

Alexandra Highet iD https://orcid.org/0000-0002-4733-2825 
John Richard Montgomery iD https://orcid.

org/0000-0001-7751-231X

Glenn Kham Wakam iD https://orcid.org/0000-0002-0950-9554

Christopher J. Sonnenday iD https://orcid.

org/0000-0002-1632-6529

Seth A. Waits iD https://orcid.org/0000-0001-6570-5288

\section{REFERENCES}

1. Notification of Enforcement Discretion for telehealth remote communications during the COVID-19 nationwide public health emergency. U.S. Department of Health \& Human Services: Health Information Privacy. https://www.hhs.gov/hipaa/for-professionals/special-topics/ emergency-preparedness/notification-enforcement-discretion-teleh ealth/index.html. Published March 19, 2020. Accessed March 22, 2020
2. John BV, Love E, Dahman B, et al. Use of telehealth expedites evaluation and listing of patients referred for liver transplantation. Clin Gastroenterol Hepatol. 2019. https://doi.org/10.1016/j. cgh.2019.12.021

3. Lee TC, Kaiser TE, Alloway R, et al. Telemedicine based remote home monitoring after liver transplantation: results of a randomized prospective trial. Ann Surg. 2019;270(3):564-572.

4. Ross K, Patzer RE, Goldberg DS, et al. Sociodemographic determinants of waitlist and post transplant survival among end-stage liver disease patients. Am J Transplant. 2017;17(11):2879-2889.

5. Latulippe K, Christine H, Dominique G. Social health inequalities and eHealth: a literature review with qualitative synthesis of theoretical and empirical studies. J Med Internet Res. 2017;19(4):e136. 